Research Article

\title{
Lump Solutions of a Nonlinear PDE Combining with a New Fourth-Order Term $D_{x}^{2} D_{t}^{2} *$
}

\author{
Liqin Zhang $\mathbb{D}^{1,2}$ Wen-Xiu Ma $\mathbb{D}^{2,3,4,5,6,7}$ and Yehui Huang $\mathbb{D}^{2,8}$ \\ ${ }^{1}$ College of Information Science and Artificial Intelligence, Xiamen Institute of Technology, Xiamen 361021, Fujian, China \\ ${ }^{2}$ Department of Mathematics and Statistics, University of South Florida, Tampa, FL 33620, USA \\ ${ }^{3}$ Department of Mathematics, Zhejiang Normal University, Jinhua, 321004 Zhejiang, China \\ ${ }^{4}$ Department of Mathematics, King Abdulaziz University, Jeddah 21589, Saudi Arabia \\ ${ }^{5}$ School of Mathematics, South China University of Technology, Guangzhou 510640, China \\ ${ }^{6}$ College of Mathematics and Systems Science, Shandong University of Science and Technology, Qingdao, 266590 Shandong, China \\ ${ }^{7}$ International Institute for Symmetry Analysis and Mathematical Modelling, Department of Mathematical Sciences, North- \\ West University, Mafikeng Campus, Private Bag X2046, Mmabatho 2735, South Africa \\ ${ }^{8}$ School of Mathematics and Physics, North China Electric Power University, Beijing 102206, China
}

Correspondence should be addressed to Liqin Zhang; aqin98@163.com and Wen-Xiu Ma; mawx@cas.usf.edu

Received 9 November 2019; Accepted 9 December 2019; Published 1 February 2020

Academic Editor: Laurent Raymond

Copyright (C) 2020 Liqin Zhang et al. This is an open access article distributed under the Creative Commons Attribution License, which permits unrestricted use, distribution, and reproduction in any medium, provided the original work is properly cited.

A nonlinear PDE combining with a new fourth-order term $D_{x}^{2} D_{t}^{2}$ is studied. Adding three new fourth-order derivative terms and some second-order derivative terms, we formulate a combined fourth-order nonlinear partial differential equation, which possesses a Hirota's bilinear form. The class of lump solutions is constructed explicitly through Hirota's bilinear method. Their dynamical behaviors are analyzed through plots.

\section{Introduction}

In the theory of differential equations, one of the fundamental problems is the Cauchy problem to determine a solution of a differential equation satisfying what are known as initial data. The classical approaches, such as Laplace's method and Fourier transform method, were developed for solving Cauchy problems for linear ordinary differential equations and for linear partial differential equations. In modern soliton theory, the isomonodromic transform method and the inverse scattering transform method have been created for handling Cauchy problems for nonlinear ordinary and partial differential equations, respectively $[1,2]$. One of the most exciting and extremely active areas of research investigation arises on the study of exact solution and the related issue of the construction of solutions to a wide class of nonlinear equations.

Exact solutions of partial differential equations describe significant mathematical and physical phenomena. A soliton solution is an exact solution determined by exponentially localized functions, which localized in all directions both in time and in space. A lump solution is also a kind of exact solutions of partial differential equations, obtained from soliton theory by taking long wave limits [1]. Nevertheless, a lump solution is localized in all directions just in space. In addition, it is well known that interaction solutions between lump solutions and soliton solutions allow to describe more nonlinear phenomena [3]. However, the interaction properties are rarely discussed because the involved mathematical computation is much more complicated. 
Generally, through a depended variable transformation, a partial differential equation can be mapped into a Hirota's bilinear form.

$$
P\left(D_{x}, D_{y}, D_{t}\right) f \cdot f=0,
$$

where $P$ is a polynomial, and $D_{x}, D_{y}, D_{t}$ are the Hirota bilinear derivatives [4], defined by

$$
\begin{aligned}
D_{x}^{l}, D_{y}^{n}, D_{t}^{m} f(x, y, t) \cdot g(x, y, t) \\
=\left(\frac{\partial}{\partial x}-\frac{\partial}{\partial x^{\prime}}\right)^{l}\left(\frac{\partial}{\partial y}-\frac{\partial}{\partial y^{\prime}}\right)^{n}\left(\frac{\partial}{\partial t}-\frac{\partial}{\partial t^{\prime}}\right)^{m} f(x, y, t) \\
\left.\quad \cdot g\left(x^{\prime}, y^{\prime}, t^{\prime}\right)\right|_{x^{\prime}=x, y^{\prime}=y, t^{\prime}=t} .
\end{aligned}
$$

When $f$ solves (2), it presents the $N$-soliton solution in $(2+1)$-dimensions to the corresponding PDE under the transformation $u=2(\ln f)_{x x}$ :

$$
f=\sum_{\mu=0,1} \exp \left(\sum_{i=1}^{N} \mu_{i} \xi_{i}+\sum_{i<j} \mu_{i} \mu_{j} a_{i j}\right)
$$

where $\sum_{\mu=0,1}$ denotes the sum over all possibilities for $\mu_{1}, \cdots$ , $\mu_{N}$ in 0,1 , and

$$
\begin{cases}\xi_{i}=k_{i} x+l_{i} y-\omega_{i} t+\xi_{i, 0}, & 1 \leq i \leq N, \\ e^{a_{i j}}=-\frac{P\left(k_{i}-k_{j}, l_{i}-l_{j}, \omega_{j}-\omega_{i}\right)}{P\left(k_{i}+k_{j}, l_{i}+l_{j}, \omega_{j}+\omega_{i}\right)}, & 1 \leq i<j \leq N,\end{cases}
$$

with $k_{i}, l_{i}, \omega_{i}$ satisfying the dispersion relation, and $\xi_{i, 0}$ being arbitrary shifts.

As is well known, the KPI equation possesses lump solutions [5]: $u=2(\ln f)_{x x}$, where

$$
\begin{aligned}
f= & \left(a_{1} x+a_{2} y+\frac{a_{1} a_{2}^{2}-a_{1} a_{6}^{2}+2 a_{2} a_{5} a_{6}}{a_{1}^{2}+a_{5}^{2}} t+a_{4}\right)^{2} \\
& +\left(a_{5} x+a_{6} y+\frac{2 a_{1} a_{2} a_{6}-a_{2}^{2} a_{5}+a_{5} a_{6}^{2}}{a_{1}^{2}+a_{5}^{2}} t+a_{8}\right)^{2} \\
& +\frac{3\left(a_{1}^{2}-a_{5}^{2}\right)^{3}}{\left(a_{1} a_{6}-a_{2} a_{5}\right)^{2}} .
\end{aligned}
$$

The condition $a_{1} a_{6}-a_{2} a_{5} \neq 0$ guarantees the rational localisation in all directions in the $(x, y)$-plane.

In the past few decades, many researchers have studied soliton solutions, lump solutions, and other classes of solutions to integrable equations, such as the Ishimori-I equation [6], the Davey-Stewarton equation II [1], the BKP equation $[7,8]$, the three-dimensional three-wave resonant interaction [9], the KP equation with a self-consistent source [10], and so on [11-14]. Some nonintegrable equations have lump solutions as well, such as the generalized KP, SawadaKotera equations [15-17]. Moreover, various studies show the existence of interaction solutions between lumps and other kinds of exact solutions to nonlinear integrable equation $[2,18-23]$.

This paper is concerned with a nonlinear PDE combining with a new fourth-order term $D_{x}^{2} D_{t}^{2}$. Adding three new fourth-order derivative terms and some second-order derivative terms, we formulate a combined fourth-order nonlinear partial differential equation, which possesses a Hirota's bilinear form. Based on a bilinear transformation, lump solutions are obtained through symbolic computation with Maple. This new term $D_{x}^{2} D_{t}^{2}$ is the second derivative with respect to time, which really makes the calculation more difficult. It is also reflected that the structure of the solution is more complex, and we pay more attention to deal with and analyze the dispersion relation of the solution. We also exhibit threedimensional plot and contour plot profiles of these solutions and study their dynamic behaviors. Some concluding remarks are given in the final section.

\section{Bilinear Form}

We would like to consider a general combined fourth-order nonlinear equation as follows:

$$
\begin{aligned}
P(u)= & \alpha_{1}\left[3\left(u_{x} u_{t}\right)_{x}+u_{x x x t}\right]+\alpha_{2}\left[3\left(u_{x} u_{y}\right)_{x}+u_{x x x y}\right] \\
& +\alpha_{3}\left[\left(v u_{x}\right)_{x}+4 u_{t} u_{x t}+u_{x x t t}\right]+\delta_{1} u_{y t}+\delta_{2} u_{x y} \\
& +\delta_{3} u_{x x}+\delta_{4} u_{t t}+\delta_{5} u_{y y}+\delta_{6} u_{x t}=0,
\end{aligned}
$$

where $v$ is a function satisfying $v_{x}=u_{t t}$, the constants $\alpha_{i}, i=1$, 2,3 all are not zero, and the constants $\delta_{i}, i=1,2,3, \cdots, 6$ are all arbitrary. We substitute the following logarithmic transformation into (6):

$$
\begin{aligned}
& u=2(\ln f)_{x}, \\
& v=2(\ln f)_{t t},
\end{aligned}
$$

and then the equation has the following Hirota's bilinear form:

$$
\begin{aligned}
B(f)= & \left(\alpha_{1} D_{x}^{3} D_{t}+\alpha_{2} D_{x}^{3} D_{y}+\alpha_{3} D_{x}^{2} D_{t}^{2}+\delta_{1} D_{y} D_{t}+\delta_{2} D_{x} D_{y}\right. \\
& \left.+\delta_{3} D_{x}^{2}+\delta_{4} D_{t}^{2}+\delta_{5} D_{y}^{2}+\delta_{6} D_{x} D_{t}\right) f \cdot f=0 .
\end{aligned}
$$

Therefore, if $f$ solves the bilinear Equation (8), then $u=$ $2(\ln f)_{x}, v=2(\ln f)_{t t}$ will solve Equation (6), where we have the relation $P(u)=\left((B(f)) / f^{2}\right)_{x}$.

When $\alpha_{1}=1, \alpha_{2}=\alpha_{3}=0, \delta_{1}=\delta_{3}=1$, and the other $\delta_{i}$, $1 \leq i \leq 6$ are zero, we obtain an integrable extension of the Hirota-Satsuma equation [4]: 


$$
3\left(u_{x} u_{t}\right)_{x}+u_{x x x t}+u_{y t}+u_{x x}=0
$$

which has a bilinear form under the logarithmic transformation $u=2(\ln f)_{x}$ as follows:

$$
\left(D_{x}^{3} D_{t}+D_{y} D_{t}+D_{x}^{2}\right) f \cdot f=0
$$

that is called the bilinear HSI equation.

When $\alpha_{2}=1, \alpha_{1}=\alpha_{3}=0, \delta_{5}=\delta_{6}=1$, and the other $\delta_{i}$, $1 \leq i \leq 6$ are zero, we obtain a generalized CalogeroBogoyavlenskii-Schiff equation [16]:

$$
3\left(u_{x} u_{y}\right)_{x}+u_{x x x y}+u_{x t}+u_{y y}=0
$$

which has a bilinear form under the logarithmic transformation $u=2(\ln f)_{x}$ as follows:

$$
\left(D_{x}^{3} D_{y}+D_{x} D_{t}+D_{y}^{2}\right) f \cdot f=0
$$

that has lump solutions [16].

\section{Lump Solutions}

A search for positive quadratic solutions to the bilinear Equation (8) generates a class of lump solutions to Equation (6):

$$
f=\left(a_{1} x+a_{2} y+a_{3} t+a_{4}\right)^{2}+\left(a_{5} x+a_{6} y+a_{7} t+a_{8}\right)^{2}+a_{9},
$$

where $a_{i}, i=1,2,3, \cdots, 9$ are arbitrary real constants to be determined. We insert (13) into (8) and then solve the resulting algebraic system by Maple symbolic computations.

We first consider the case of $\delta_{2}=\delta_{4}=\delta_{5}=0$ for the combined nonlinear Equation (6). We obtain the following parameters:

$$
\left\{\begin{array}{l}
a_{3}=-\frac{a_{1}\left(a_{1}^{2}+a_{5}^{2}\right) \delta_{3} \delta_{6}+\left(a_{1}^{2} a_{2}+2 a_{1} a_{5} a_{6}-a_{2} a_{5}^{2}\right) \delta_{1} \delta_{3}}{\left(a_{1} \delta_{6}+a_{2} \delta_{1}\right)^{2}+\left(a_{5} \delta_{6}+a_{6} \delta_{1}\right)^{2}}, \\
a_{7}=-\frac{a_{5}\left(a_{1}^{2}+a_{5}^{2}\right) \delta_{3} \delta_{6}-\left(a_{1}^{2} a_{6}+2 a_{1} a_{2} a_{5}-a_{5}^{2} a_{6}\right) \delta_{1} \delta_{3}}{\left(a_{1} \delta_{6}+a_{2} \delta_{1}\right)^{2}+\left(a_{5} \delta_{6}+a_{6} \delta_{1}\right)^{2}}, \\
a_{9}=-\frac{b_{1} \alpha_{1}+b_{2} \alpha_{2}+b_{3} \alpha_{3}}{\left(a_{1} a_{6}-a_{2} a_{5}\right)^{2}\left[\left(a_{1} \delta_{6}+a_{2} \delta_{1}\right)^{2}+\left(a_{5} \delta_{6}+a_{6} \delta_{1}\right)^{2}\right] \delta_{1}^{2} \delta_{3}},
\end{array}\right.
$$

and all other $a_{1}$ are arbitrary. The above involved three constants $b_{i}, i=1,2,3$, are defined as follows:

$$
\left\{\begin{array}{l}
b_{1}=-3\left(a_{1}^{2}+a_{5}^{2}\right)^{2}\left[\left(a_{1}^{2}+a_{5}^{2}\right) \delta_{6}+\left(a_{1} a_{2}+a_{5} a_{6}\right) \delta_{1}\right]\left[\left(a_{1} \delta_{6}+a_{2} \delta_{1}\right)^{2}+\left(a_{5} \delta_{6}+a_{6} \delta_{1}\right)^{2}\right], \\
b_{2}=3\left(a_{1}^{2}+a_{5}^{2}\right)\left(a_{1} a_{2}+a_{5} a_{6}\right)\left[\left(a_{1} \delta_{6}+a_{2} \delta_{1}\right)^{2}+\left(a_{5} \delta_{6}+a_{6} \delta_{1}\right)^{2}\right]^{2} \\
b_{3}=3\left(a_{1}{ }^{2}+a_{5}{ }^{2}\right)^{2}\left[3\left(a_{1} a_{2}+a_{5} a_{6}\right)^{2}+\left(a_{1} a_{6}-a_{2} a_{5}\right)^{2}\right] \delta_{1}{ }^{2} \delta_{3}^{2}+3\left(a_{1}{ }^{2}+a_{5}{ }^{2}\right)^{4} \delta_{3}{ }^{2} \delta_{6}^{2}+6\left(a_{1}{ }^{2}+a_{5}{ }^{2}\right)^{3}\left(a_{1} a_{2}+a_{5} a_{6}\right) \delta_{1} \delta_{6} \delta_{3}^{2} .
\end{array}\right.
$$

Therefore, the condition guaranteeing the nonsingularity of the lump solution is $\left(a_{1} a_{6}-a_{2} a_{5}\right)^{2}\left[\left(a_{1} \delta_{6}+a_{2} \delta_{1}\right)^{2}+\right.$ $\left.\left(a_{5} \delta_{6}+a_{6} \delta_{1}\right)^{2}\right] \delta_{1}{ }^{2} \delta_{3} \neq 0$, and they should satisfy the following constraint conditions:

$$
-\frac{b_{1} \alpha_{1}+b_{2} \alpha_{2}+b_{3} \alpha_{3}}{\delta_{3}}<0
$$

Now, the transformations in (7) generate a large class of lump solutions to (6), determined by $u=2(\ln f)_{x}, v=2$ $(\ln f)_{t t}$ :

$$
\left\{\begin{array}{l}
u=\frac{4 a_{1}\left(a_{1} x+a_{2} y+a_{3} t+a_{4}\right)+4 a_{5}\left(a_{5} x+a_{6} y+a_{7} t+a_{8}\right)}{f}, \\
v=\frac{4\left(a_{3}^{2}+a_{7}^{2}\right) f-4\left[a_{1}\left(a_{1} x+a_{2} y+a_{3} t+a_{4}\right)+4 a_{5}\left(a_{5} x+a_{6} y+a_{7} t+a_{8}\right)\right]^{2}}{f^{2}} .
\end{array}\right.
$$


We take a special choice for the parameters: $\delta_{1}=1, \delta_{3}=1$, $\delta_{6}=1, \alpha_{1}=\alpha_{2}=\alpha_{3}=1, a_{1}=2, a_{2}=-1, a_{3}=224 / 785, a_{4}=5$, $a_{5}=-2, a_{6}=-30, \quad a_{7}=-8 / 785, \quad a_{8}=6, \quad a_{9}=227007188 /$ 660185 , under which by $f(13),(14)$, and (15) will present the lump solution to (6).

$$
\begin{aligned}
f= & \left(2 x-y-\frac{224}{785} t+5\right)^{2}+\left(-2 x-30 y-\frac{8}{785} t+6\right)^{2} \\
& +\frac{227007188}{660185}
\end{aligned}
$$

We plot the graphs of this solution in Figures 1 and 2 .

By calculating, we find that the lump solution reaches the peak when $t=0$. As time goes on, the waves moving forward reach the same amplitude at other points. The results provide new insights into the generation of the large amplitude waves and thus are useful in the application or prevention of the large amplitude waves in the propagation of many important nonlinear physical situations. Next, we will consider the application of these results to physical theory and experiments and its relation with the initial boundary value problem is also considered.

We secondly consider the case of $\delta_{1}=\delta_{5}=\delta_{6}=0$ for the combined nonlinear Equation (6). We obtain the following parameters:

$$
\left\{\begin{array}{l}
a_{7}=b \\
a_{3}=\frac{2 a_{1} a_{5} \delta_{3}+\left(a_{1} a_{6}+a_{2} a_{5}\right) \delta_{2}}{2 b \delta_{4}}, \\
a_{9}=-\frac{\left(a_{1}^{2}+a_{5}^{2}\right)\left(c_{1} \alpha_{1}+c_{2} \alpha_{2}+c_{3} \alpha_{3}\right)}{\left(2 a_{1} a_{5} \delta_{3}+\delta_{2}\left(a_{1} a_{6}+a_{2} a_{5}\right)\right)^{2}+4 b^{2} a_{1} a_{2} \delta_{2} \delta_{4}+4 b^{2} a_{1}^{2} \delta_{3} \delta_{4}},
\end{array}\right.
$$

and all other $a_{1}$ are arbitrary. The above involved three constants $b_{i}, i=1,2,3$ are defined as follows:

$$
\left\{\begin{array}{l}
c_{1}=3\left[-a_{1}\left(a_{1} a_{6}+a_{2} a_{5}\right) \delta_{2}-2 a_{1}{ }^{2} a_{5} \delta_{3}+2 b^{2} a_{5} \delta_{4}\right] b \delta_{4}, \\
c_{2}=6\left(a_{1} a_{2}+a_{5} a_{6}\right) b^{2} \delta_{4}^{2}, \\
c_{3}=2\left[\left(a_{1}{ }^{2}-3 a_{5}{ }^{2}\right) b^{2} \delta_{3} \delta_{4}+\left(a_{1} a_{2}-3 a_{5} a_{6}\right) b^{2} \delta_{2} \delta_{3}+\left(2 a_{1} a_{5} \delta_{3}+\left(a_{1} a_{6}+a_{2} a_{5}\right) \delta_{2}\right)^{2}\right] .
\end{array}\right.
$$

Therefore, the condition guaranteeing the nonsingularity of the lump solution is as follows:

$\left(2 a_{1} a_{5} \delta_{3}+\delta_{2}\left(a_{1} a_{6}+a_{2} a_{5}\right)\right)^{2}+4 b^{2} a_{1} a_{2} \delta_{2} \delta_{4}+4 b^{2} a_{1}{ }^{2} \delta_{3} \delta_{4} \neq 0$,

and they should satisfy the following constraint conditions: $a_{9}>0$.

We take a special choice for the parameters: $\delta_{2}=1$, $\delta_{3}=1, \delta_{4}=1, \alpha_{1}=\alpha_{2}=\alpha_{3}=1, a_{1}=-1, a_{2}=11, a_{3}=10(1 /$ $(\sqrt{-5+5 \sqrt{5}})), \quad a_{4}=1, a_{5}=-2, a_{6}=2, \quad a_{7}=1 /(\sqrt{-5+5 \sqrt{5}})$, $a_{8}=1, \quad a_{9}=-1 / 2((6 \sqrt{-5+5 \sqrt{5}} \sqrt{5}+55 \sqrt{5}-135) /(\sqrt{5}-3))$, and

$$
\begin{aligned}
f= & \left(10 \frac{t}{\sqrt{-5+5 \sqrt{5}}}-x+11 y+1\right)^{2} \\
& +\left(\frac{t}{\sqrt{-5+5 \sqrt{5}}}-2 x+2 y+1\right)^{2} \\
& -\frac{6 \sqrt{-5+5 \sqrt{5}} \sqrt{5}+55 \sqrt{5}-135}{2 \sqrt{5}-6},
\end{aligned}
$$

under which $f$ by (13), (19), and (20) will present the lump solution to (6), determined by $u=2(\ln f)_{x}, v=2(\ln f)_{t t}$.

$$
\left\{\begin{array}{l}
u=\frac{-4(10(t /(\sqrt{-5+5 \sqrt{5}}))-x+11 y+1)-8((t / \sqrt{-5+5} \sqrt{5})-2 x+2 y+1)}{f}, \\
v=\frac{2 f_{t t} f-2\left[f_{t}\right]^{2}}{f^{2}} .
\end{array}\right.
$$

We plot the graphs of this solution in Figures 3 and 4 .

We have presented lump solutions to a combined fourthorder nonlinear partial differential equation by means of the
Hirota bilinear formulation. The solutions have been depicted for special values of the parameters and three different values of $t$. Figure 3 shows lump wave. By calculating, we 


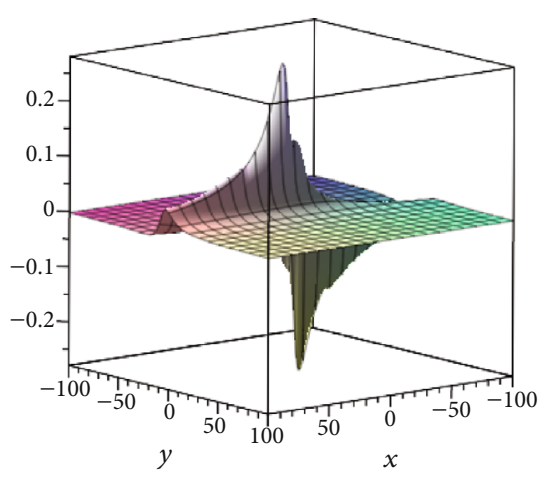

(a)

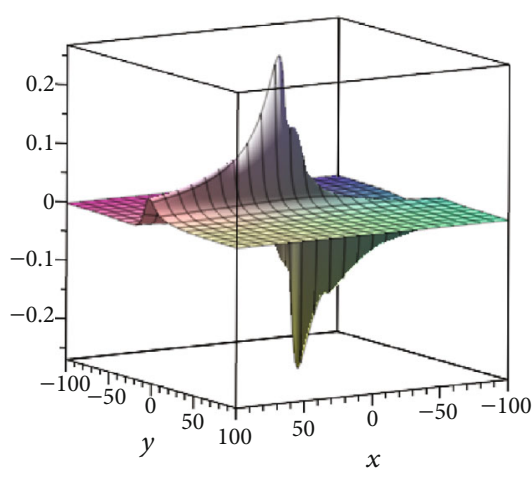

(b)

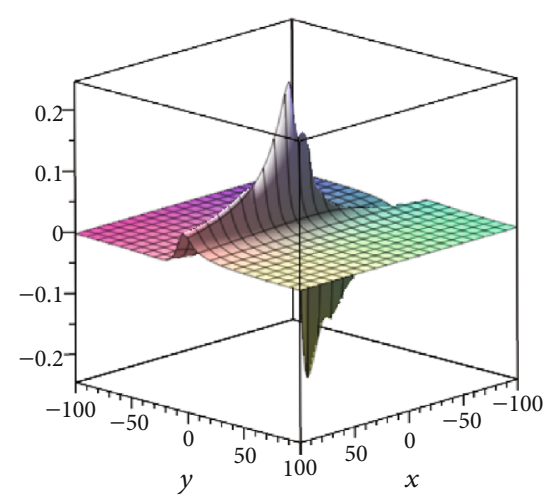

(c)

Figure 1: (Color online) soliton solution $u$ with $t=0,15,30$ : 3D plots.

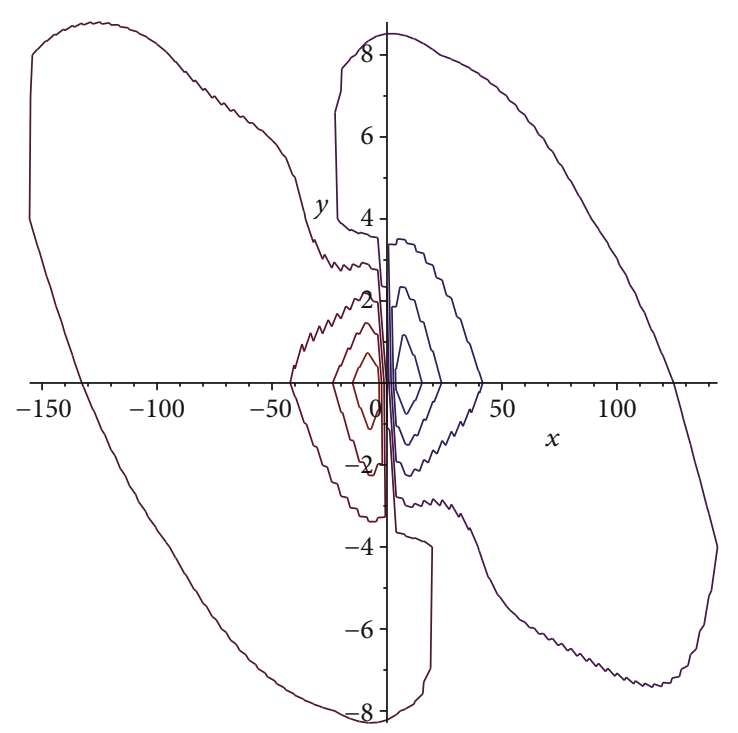

(a)

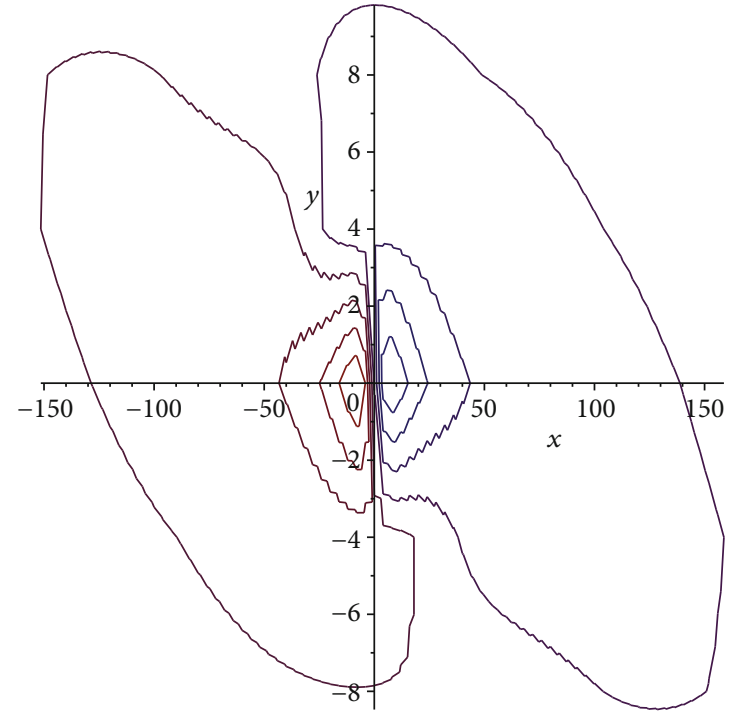

(b)

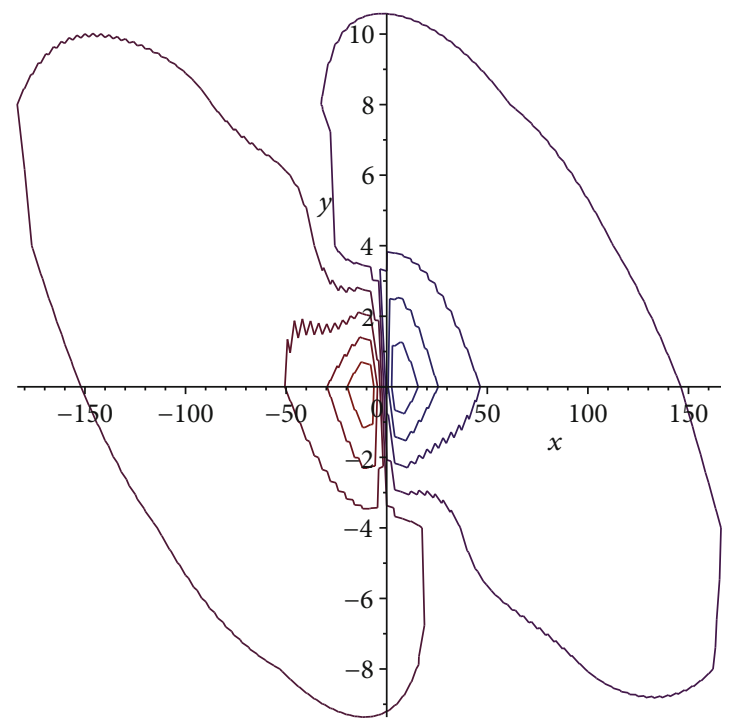

(c)

FIGURE 2: (Color online) soliton solution $u$ with $t=0,10,30$ : contour plots. 


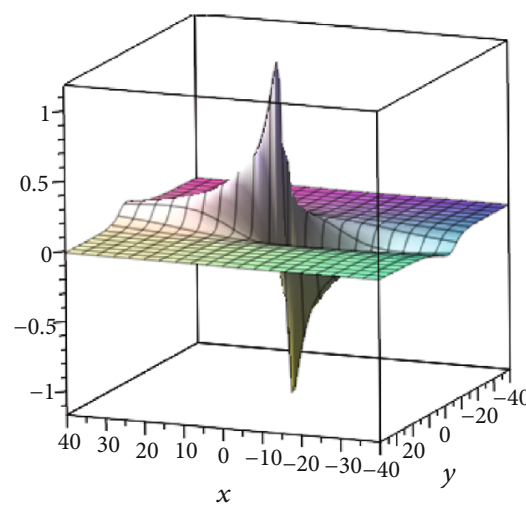

(a)

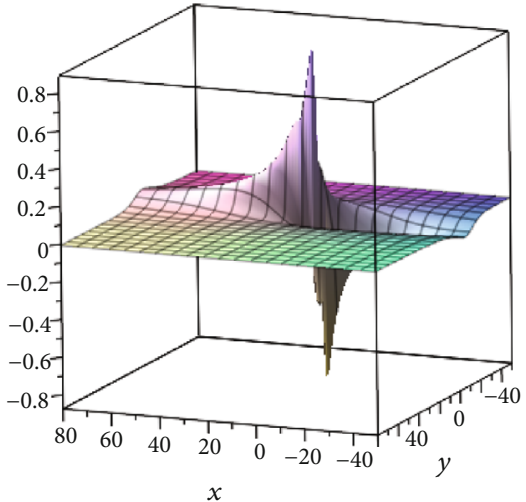

(b)

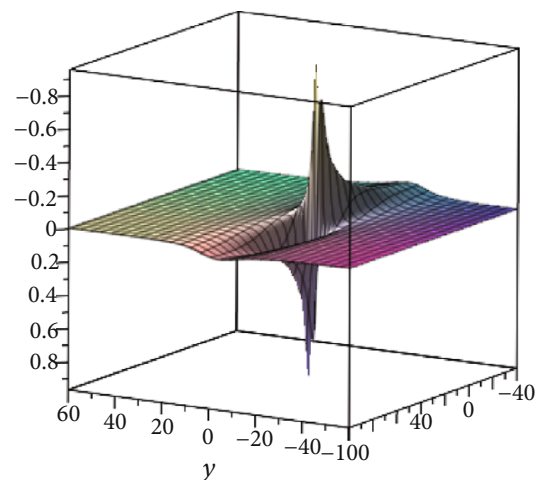

(c)

FIgURE 3: (Color online) soliton solution $u$ with $t=0,15,30$ : 3D plots.

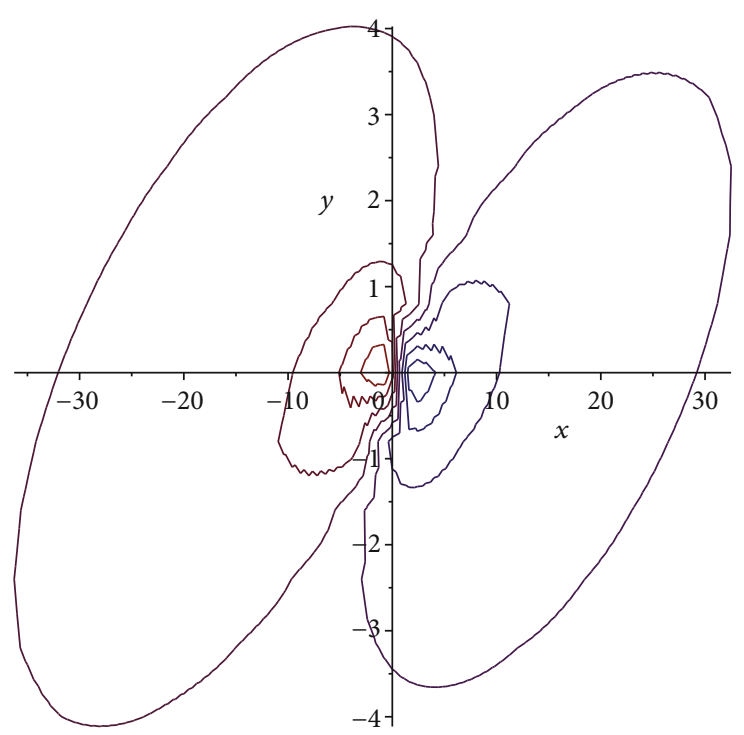

(a)

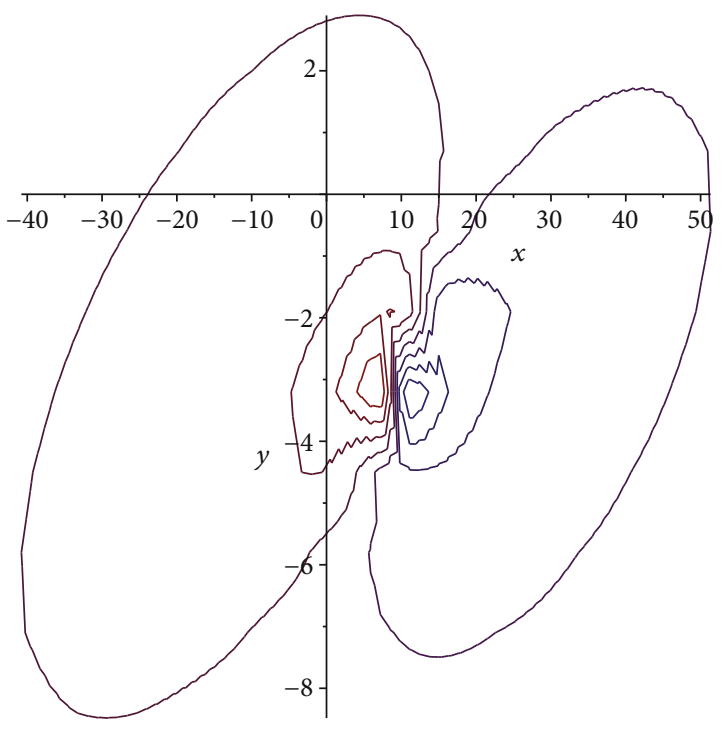

(b)

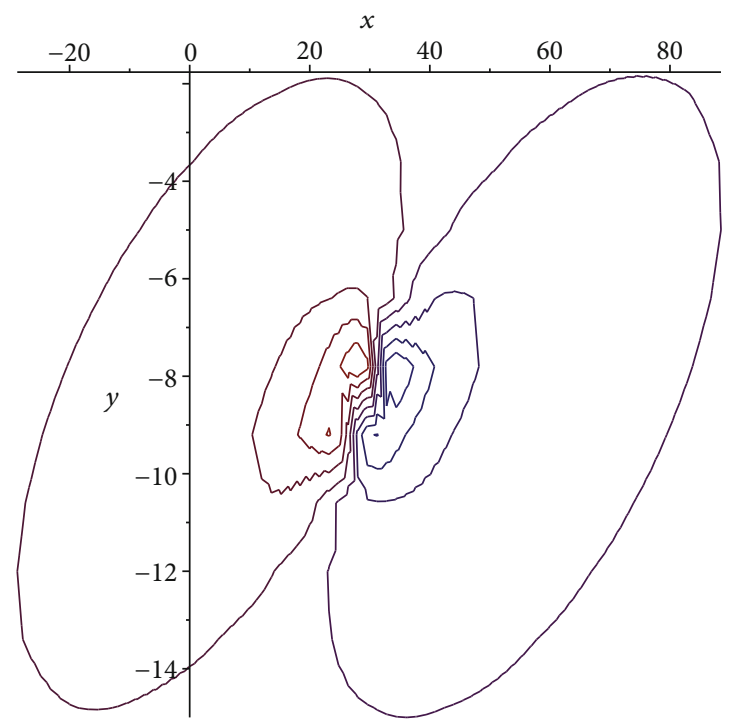

(c)

FIGURE 4: (Color online) soliton solution $u$ with $t=0,10,30$ : contour plots. 
find that the lump solution has a peak when $t=0$. As time goes on, the waves moving forward reach the same amplitude at other points.

\section{Conclusion and Remarks}

In this paper, we have considered a combined fourth-order nonlinear partial differential equation. We have worked out a class of lump solutions by Hirota's bilinear method. It is important to remark that the three nonlinear terms can be merged together into the considered nonlinear model. It is worth pointing out that the term $D_{x}^{2} D_{t}^{2}$ makes the calculation more difficult. It is also reflected that the structure of the solution is more complex. No one has considered it over before. All the above results offer us abundant new exact solutions, which enrich the existing theories of solutions [24-29] to equations, and add valuable insights into soliton solutions and dromion-type solutions, developed through various powerful solution techniques including the Hirota perturbation approach, the Riemann-Hilbert approach, the Wronskian technique, symmetry reductions, and symmetry constraints. It is also interesting to research for lump and interaction solutions to other generalized bilinear differential equations [30-34]. The research to establish a fundamental theory of lump and interaction solutions for PDEs deserve our further effort.

\section{Data Availability}

The data used to support the findings of this study are available from the corresponding authors upon request.

\section{Conflicts of Interest}

The authors declare that they have no conflicts of interest.

\section{Acknowledgments}

The work was supported by the National Natural Science Foundation of China (Grant No. 11672074), the NSF under Grant No. DMS-1664561, and also supported by the Fundamental Research Funds for the Central Universities (Grant No. 2016MS63).

\section{References}

[1] J. Satsuma and M. J. Ablowitz, "Two-dimensional lumps in nonlinear dispersive systems," Journal of Mathematical Physics, vol. 20, no. 7, pp. 1496-1503, 1979.

[2] T. C. Kofane, M. Fokou, A. Mohamadou, and E. Yomba, "Lump solutions and interaction phenomenon to the thirdorder nonlinear evolution equation," The European Physical Journal Plus, vol. 132, no. 11, pp. 465-473, 2017.

[3] W. X. Ma and Y. You, "Solving the Korteweg-de Vries equation by its bilinear form: Wronskian solutions," Transactions of the American Mathematical Society, vol. 357, no. 5, pp. 1753-1779, 2005.

[4] R. Hirota, The Direct Method in Soliton Theory, Cambridge University Press, 2004.
[5] W. X. Ma, "Lump solutions to the Kadomtsev-Petviashvili equation," Physics Letters A, vol. 379, no. 36, pp. 1975-1978, 2015.

[6] K. Imai, "Dromion and lump solutions of the Ishimori-I equation," Progress in Theoretical Physics, vol. 98, no. 5, pp. 1013 1023, 1997.

[7] C. R. Gilson and J. J. C. Nimmo, "Lump solutions of the BKP equation,” Physics Letters A, vol. 147, no. 8-9, pp. 472-476, 1990.

[8] J. Y. Yang and W. X. Ma, "Lump solutions to the BKP equation by symbolic computation," International Journal of Modern Physics B, vol. 30, no. 28n29, article 1640028, 2016.

[9] D. J. Kaup, “The lump solutions and the Bäcklund transformation for the three-dimensional three-wave resonant interaction," Journal of Mathematical Physics, vol. 22, no. 6, pp. 1176-1181, 1981.

[10] X. Yong, W. X. Ma, Y. Huang, and Y. Liu, "Lump solutions to the Kadomtsev-Petviashvili I equation with a self-consistent source," Computers \& Mathematics with Applications, vol. 75, no. 9, pp. 3414-3419, 2018.

[11] W. X. Ma, Z. Qin, and X. Lü, "Lump solutions to dimensionally reduced $\boldsymbol{p}$-gKP and $\boldsymbol{p}$-gBKP equations," Nonlinear Dynamics, vol. 84, no. 2, pp. 923-931, 2016.

[12] W. X. Ma, X. Yong, and H. Q. Zhang, "Diversity of interaction solutions to the (2+1)-dimensional Ito equation," Computers \& Mathematcs with Applications, vol. 75, no. 1, pp. 289-295, 2018.

[13] W. X. Ma and Y. Zhou, "Lump solutions to nonlinear partial differential equations via Hirota bilinear forms," Journal of Differential Equations, vol. 264, no. 4, pp. 2633-2659, 2018.

[14] J. B. Zhang and W. X. Ma, "Mixed lump-kink solutions to the BKP equation," Computers \& Mathematics with Applications, vol. 74, no. 3, pp. 591-596, 2017.

[15] H. Q. Zhang and W. X. Ma, "Lump solutions to the $(2+1)$ -dimensional Sawada-Kotera equation," Nonlinear Dynamics, vol. 87, no. 4, pp. 2305-2310, 2017.

[16] S. T. Chen and W. X. Ma, "Lump solutions to a generalized Bogoyavlensky-Konopelchenko equation," Frontiers of Mathematics in China, vol. 13, no. 3, pp. 525-534, 2018.

[17] W. X. Ma, "A search for lump solutions to a combined fourthorder nonlinear PDE in (2+1)-dimensions," Journal of Applied Analysis and Computation, vol. 9, pp. 1319-1332, 2019.

[18] W. X. Ma, Y. Zhou, and R. Dougherty, "Lump-type solutions to nonlinear differential equations derived from generalized bilinear equations," International Journal of Modern Physics $B$, vol. 30, no. 28n29, article 1640018, 2016.

[19] J. Y. Yang, W. X. Ma, and Z. Qin, "Lump and lump-soliton solutions to the $(2+1)$-dimensional Ito equation," Analysis and Mathematical Physics, vol. 8, no. 3, pp. 427-436, 2018.

[20] W. X. Ma, "Lump-type solutions to the $(3+1)$-dimensional Jimbo-Miwa equation," International Journal of Nonlinear Sciences and Numerical Simulation, vol. 17, no. 7-8, pp. 355-359, 2017.

[21] J. Y. Yang and W. X. Ma, "Abundant lump-type solutions of the Jimbo-Miwa equation in (3+1)-dimensions," Computers \& Mathematics with Applications, vol. 73, no. 2, pp. 220-225, 2017.

[22] Y. Tang, S. Tao, and Q. Guan, "Lump solitons and the interaction phenomena of them for two classes of nonlinear evolution equations," Computers \& Mathematcs with Applications, vol. 72, no. 9, pp. 2334-2342, 2016. 
[23] H. Q. Zhao and W. X. Ma, "Mixed lump-kink solutions to the KP equation," Computers \& Mathematcs with Applications, vol. 74, no. 6, pp. 1399-1405, 2017.

[24] B. Konopelchenko and W. Strampp, "The AKNS hierarchy as symmetry constraint of the KP hierarchy," Inverse Problems, vol. 7, no. 2, pp. L17-L24, 1991.

[25] B. Dorizzi, B. Grammaticos, A. Ramani, and P. Winternitz, "Are all the equations of the Kadomtsev-Petviashvili hierarchy integrable?," Journal of Mathematical Physics, vol. 27, no. 12, pp. 2848-2852, 1986.

[26] X. Y. Li, Q. L. Zhao, Y. X. Li, and H. H. Dong, "Binary Bargmann symmetry constraint associated with $3 \times 3$ discrete matrix spectral problem," Journal of Nonlinear Science and Applications, vol. 8, no. 5, pp. 496-506, 2015.

[27] Q. L. Zhao and X. Y. Li, "A Bargmann system and the involutive solutions associated with a new 4-order lattice hierarchy," Analysis and Mathematical Physics, vol. 6, no. 3, pp. 237-254, 2016.

[28] H. Dong, Y. Zhang, and X. Zhang, "The new integrable symplectic map and the symmetry of integrable nonlinear lattice equation," Communications in Nonlinear Science and Numerical Simulation, vol. 36, pp. 354-365, 2016.

[29] X. Y. Li and Q. L. Zhao, "A new integrable symplectic map by the binary nonlinearization to the super AKNS system," Journal of Geometry and Physics, vol. 121, pp. 123-137, 2017.

[30] W. X. Ma, "Generalized bilinear differential equations," Studies in Nonlinear Sciences, vol. 2, pp. 140-144, 2011.

[31] W. X. Ma, "Bilinear equations, Bell polynomials and linear superposition principle," Journal of Physics: Conference Series, vol. 411, article 012021, 2013.

[32] H. An, D. Feng, and H. Zhu, "General M-lump, highorder breather and localized interaction solutions to the 2+1-dimensional Sawada-Kotera equation," Nonlinear Dynamics, vol. 98, no. 2, pp. 1275-1286, 2019.

[33] L. Chen, J. Chen, and Q. Chen, "Mixed lump-soliton solutions to the two-dimensional Toda lattice equation via symbolic computation," Nonlinear Dynamics, vol. 96, no. 2, pp. 15311539, 2019.

[34] D. Feng, H. An, H. Zhu, and Y. Zhao, "The synchronization method for fractional-order hyperchaotic systems," Physics Letters A, vol. 383, no. 13, pp. 1427-1434, 2019. 